\title{
On Applied Skills of Character Design in Graphic Design
}

\author{
Yang Liuyi \\ Modern Art College, Jianghan University, Wuhan, China \\ 969021787@qq.com
}

Keywords: Character Design, Graphic Design, Applied Skills

\begin{abstract}
Graphic design is an academic study which reflect aesthetics, and it is also an important part of visual communication. Font design is a form of composition that apply in the graphic design. Font is a symbol formed in human daily life that can express some kind of topics and substance, and those symbol not only can convey about ideas and thoughts of human but also can communicate about emotion and information between people. No matter in what visual media, font and graphic are always been two biggest part of it. In graphic design there is a only way to strengthen the effect of visual communication is using creative imagination rationally to design the font, and that would make project express more information and get better value in aesthetic.
\end{abstract}

\section{Introduction}

In graphic design, the application of graphics, word, illustrations, color and other elements constitutes various forms of design. Word is the main factor in graphic design constitutes and is an important tool for the exchange of information. Character design means overall arrange word carefully according to visual law. In any media, it not only is the carrier of language and information, but also has intuitive transmission; it not only is able to convey the author's effective intent, but also is the elements of visual communication for expressing the design theme and concept; it is also the induction and tips conveyed by the graphic work. Enhance visual communication effects, and improve the demands of pursuit force and give the correct aesthetic value to design. In the role of transmission of information of graphic design, it plays a very important role. Word expresses abstract meaning with its physical beauty, giving a strong artistic appeal. The author will explain specifically the applied skills of character design in graphic design in the following word.

\section{Principle of Character Design}

The objects involved in graphic design are more widely, and they are logo design, advertising design, corporate identity design and packaging design, etc. For these objects, word is an indispensable part, and even a considerable number of designs are formed by word purely. Character design is a process of artistic creation, and is a visual means of communication to regroup and rearrange elements (word, graphics and color, etc.) in graphic design. Selecting font, size, collocation, and layout are not arbitrary, and character design must be created for specific purpose ${ }^{[1]}$. Whether the Character design is arranged reasonably affects the layout of visual communication effects directly.

\section{(1) The Legibility and Readability of Word}

Word is not only the symbol for inheriting civilization, but also a modeling language. There are three levels of word. They are the word written on the surface, the word in the linguistics and the word inspiring artistic imagination of people. Legibility refers to the distinguishing extent of a single word. The word is designed to show them clearly and concisely, and convey design content to the audience accurately and vividly. Legibility focuses on clarity of information transmission, while readability can stimulate people's interest in reading ${ }^{[2]}$. Therefore, in the character design, only change the strokes regularly to make the word creating more creative and expressive, and thus creating the works with vitality and power. And then stimulate people's artistic appeal and also convey the idea of the designer more effectively, express the design theme and leave striking and unforgettable impressions. 


\section{(2) The Order of Character Design}

In character design, the refreshing emotional and rational order should be integrated, and give the audience a kind of enjoyment beyond word and image. Overall factors should be taken into account, do not form the conflict in the visual order. In order to make the design more holistic and have more sense of order, character design should be coordinated with other elements, otherwise it will be disorganized, and the demands of work and the atmosphere are likely to be destroyed. Only we refine similarity in works, take appropriate transformation and deformation can make the whole design harmonious, thereby generating the overall decorative effects with common features.

\section{(3) Creative Thinking of Character Design}

The character design should not only meets the requirements of the design theme, but also have a distinct personality and originality, that is, character design can not only reveal the nature of the graphic design theme, but also come up with new and constructive ideas ${ }^{[4]}$. During character design, analyze configuration rules and graphic symbolized characteristics, use similarity of fonts to group different forms and create word with rich charm, so that their design and style can stimulate the aesthetic pleasure of audience and make a finishing touch to graphic design.

\section{(4) Visual Beauty of Character Design}

Under the influence of the environment, people have a certain aesthetic, which plays a role not only in information transmission, but also in enhancing the visual aesthetic. The structure of the word is to seek balance and show the beauty of order. Only grasp meeting point of real life and character design, and find and understand, can we create the works with ingenious combination and graceful shape. Only touch people's soul and bring good visual effects can audience be memorable, and make the word achieve the dual purpose of information transmission and visual aesthetic.

\section{(5) The Relationship between Word and Other Elements}

Word, graphics and color are the basic elements of graphic design. The relationships among word, graphics and color should be designed carefully so that they achieve the purposes of reason and coordination. Through the design and arrangement of master-slave relationship of elements, visual order and spatial level, strengthen the visual process and highlight the design theme. Therefore, the core of graphic design lies in whether relationships among word, graphics and color can be handled reasonably and flexibly.

\section{The Combination of Word}

Whether the word is combined well or bad has a direct impact on the visual communication effect of layout. In visual communication design, in addition to using basic fonts, we often use creative fonts, and sometimes we need foreign language fonts ${ }^{[5]}$. When using several different fonts simultaneously, they should be based on the design theme and creative composition, cooperate and coordinate mutually to obtain the appropriate combining form. Different combinations of fonts can convey different visual effects and give rise to different emotion and psychological reactions. Improper combination of font is likely to cause congestion and disco-ordination, is not conducive to subject content appeals of the entire design work, and it is difficult to produce good visual impact. In order to get a good effect of font combination, it is necessary to identify common principles among different fonts, for different opposing factors to be combined harmoniously, under the premise of recognition and understanding, select contrasting factors from the aspects of style, density, orientation, brightness, etc. there is the odd flat, and there are common in odd, rational sensibility, and achieve the overall coordination.

(1) The Visual Process of Character Design

When people observe the external image information, the visual feeling is formed. People's visions have a habitual direction and order, thus forming the visual process, that is, the invisible line in gradually design works people read. Therefore, the way of combining word needs to meet people's visual processes of psychological feelings and guide people's attention by design appeal to get the best information initiatively. The research shows that people's eyes are not reading word for word in smooth and linear manner, but a series of activities. Within a certain range, the sight flow to the middle-upper and left-upper. The upper part gives people a sense of ease, which is the strongest 
part of the visual flow; the lower part makes people feel smooth and depressed.

(2) The Unified Basic Design

Each graphic design has its own unique style. Under this premise, the character design is conducting creative activities of form, strokes, structure, shape and creative content of word. Designers should integrate thematic content and word content, etc. and seize the moment of thoughts feelings and fit imagination of art and create awareness into the design to create a novel, beautiful and unique fonts. Character design has charm of being alike in spirit and conveying feelings and gives the relaxed, serious or lyrical artistic feelings. But they must be satisfied with a large graphic design theme, and form a unified feelings keynote. Thus, the entire design will meet the visual beauty, be in line with people's psychological appreciation to enhance information transmission and achieve the aesthetic purposes.

\section{(3) Three-dimensional Space and Negative Space}

In the character design, three-dimensional modeling is quite common. It uses the principle of perspective and shape three-dimensional word with height, width and depth or character design with space environment on the plane. Three-dimensional modeling is through parallel perspective, poly-point perspective, standard stereo, shadows, reflections and other performance practices, thus producing a visual conflict with each other and giving the audience different visual feelings. In addition, the space occupied by the fonts themselves is called negative space, that is, the surrounding black area and the pitch left by character design. Whether the design of combining words is successful depends largely on whether the use of negative space is appropriate.

\section{The Form Design of the Word}

There are different visual feelings of different fonts in graphic design, such as condensed word with the feelings of flow up and down, extended word with the feelings of flow left and right and Italic with the dynamic feelings of forward or diagonal flow ${ }^{[6]}$. Therefore, in the eyes of designers, there are rich changes of shape. Only accurately grasp the rule of change and use these changes skillfully, we can achieve the desired design effects. In modern character design, font size, font and color are the main expression of word. The key for from design of word is that take varieties of factors for forming word into overall consideration, use aesthetic techniques to design carefully and achieve the purposes of showing emphasis, harmonious layout and enhancing the visual feeling of the audience.

(1) The role of font and font size change is not only the role of design in the layout composition, but also for strengthening the content of the master and slave relations play a very important role, for maximum visual impact. First, size composition and layout design should be based on the theme of the performance needs; secondly, in the design technique that should be based on themes and the entire layout of the arrangement to adjust the font size; Again, font contrasting design in order to improve the word of the striking degree.

\section{(2) Font Design}

Font is the soul of the word. The font design can inject the soul into the word. In the early font design, the main task of font is to create new fonts and transform the old fonts. How to select the appropriate fonts from wide varieties of fonts and apply them into the graphic design is the issue considered by the graphic designers repeatedly.

(3) The Design of the Effects of Word

The effects of word are the additional external forms of words. The design of the effects of word includes: color, variant and free arrangement, etc. In today's graphic design, the effects of word are mostly realized through computer design, but the design and the main body of creation at the conception stage are accomplished by designers.

\section{Summary}

In short, in today's rapidly developing society, the words have become the most direct and most effective element for visual communication. This function of the fonts makes them play a pivotal 
role in competition in the international market. Therefore, only use reasonable imaginative creation for character design in graphic design, we can enhance the effectiveness of visual communication, improve the artistic appeal of the works designed and give the works designed a better information transmission and aesthetic value.

\section{References}

[1] Mao Xi. The Design and Layout of the Font [M]. Shanghai People's Fine Arts Publishing House, 2007.

[2] Zhao Zhenqian. Font Design [M]. Zhengzhou: Henan Science and Technology Press, 2007.

[3] Feng Minggao. Layout Design [M]. Changsha: Hunan University Press, 2004.

[4] Dang Sheng. The Design of Fonts, Symbols and Layout [M]. Xi'an: Shanxi People's Fine Arts Publishing House, 2000.

[5] David, Zhu Li. What is Typo Graphy? [M]. Beijing: China Youth Publishing House, 2010.

[6] Zhao Zijiang. The Art of Graphic Design [M]. Beijing: China Machine Press, 2005. 